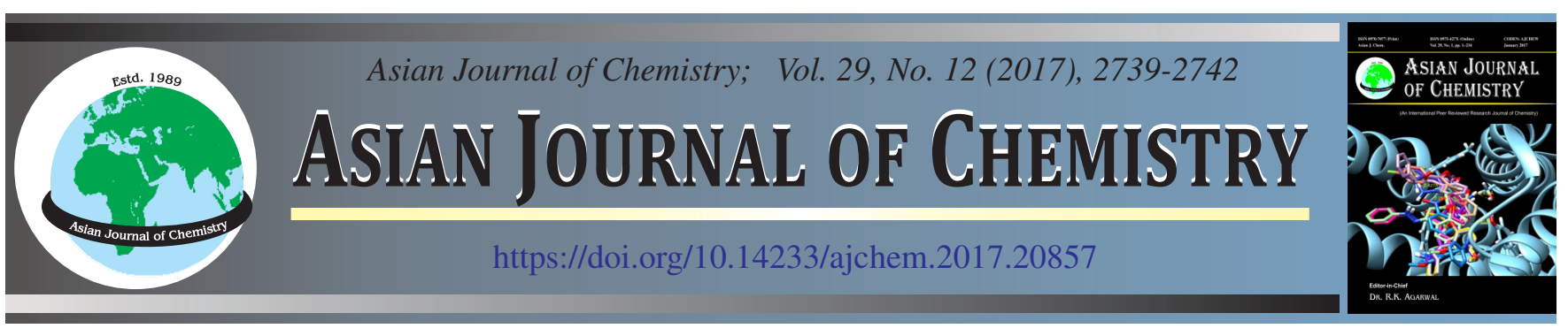

\title{
Theoretical Studies of Some Polymeric-Cu(II)-Pyrimidine-Carboxylate Ligands: Crystal Parameters and $J$ Exchange Coupling
}

\author{
RaghdaA A. Massoud, Nicholas Lees-Gayed and Mohamed A. Makhyoun*
}

Chemistry Department, Faculty of Science, Alexandria University, Alexandria, 21525 Egypt

*Corresponding author: E-mail: makhyoun2@yahoo.com

Keywords: DFT method, Spin exchange coupling, Crystal parameters.

\section{INTRODUCTION}

Polymeric pyrimidines containing copper(II) complexes are well known in different formulations. They exhibit a variety of magnetic interactions. It seems that the two nitrogen atoms of the pyrimidine ring are oriented favourably to form coordinate bonds with an infinite $1 \mathrm{D}$ chain. In the presence of other ligands, 2D layers are also possible. For example, the simple dinitrato polymer shows ferromagnetic coupling between the copper(II) centers, a 2D infinite layered structure with bridging pyrimidine ligands and $J=+0.62 \mathrm{~cm}^{-1}[1]$.

The complex polymer $\left[\mathrm{Cu}_{2}(\text { oxen })(\text { pym })_{2}\right]_{\mathrm{n}}\left(\mathrm{ClO}_{4}\right)_{2 \mathrm{n}}$, where oxen and pym are the N,N'-(2-aminoethyl)oxamide dianion and pyrimidine respectively, was synthesized and characterized by magnetic measurements [2]. This compound consists of layers of a two-dimensional network arrayed along the a axis. Within the layer, trans-oxamido-bridged copper(II) dimers connected by pyrimidine in an asymmetric fashion spread out over the bc plane to form 2D infinite networks. The magnetic study of the compound showed a strong antiferromagnetic exchange interaction with $J=-582 \mathrm{~cm}^{-1}$. The copper(II) atoms are separated by about $5.2 \AA$.

Another complex, $\mathrm{Cu}\left(\mathrm{NO}_{3}\right)_{2}\left(\mathrm{H}_{2} \mathrm{O}\right)_{2}(\mathrm{pym})_{2}$ showed antiferromagnetic interactions at extremely low temperatures [3]. In this compound the pyrimidine bridges the copper ions to form one-dimensional coordination chains. Formation of coordination polymers allows the appearance of the magnetic interactions. The details of the coordination of the bridging organic moieties should also play an important role in the magnetic properties. Magnetic measurements show that it is a good example of a uniform $S=1 / 2$ antiferromagnetic Heisenberg chain with exchange parameter

$$
J=-12.5 \mathrm{~cm}^{-1} \text {. }
$$

The pyrimidine complexes under theoretical investigation here are compounds $(\mathbf{1})$ and (2). Compound $\mathbf{1}$ is [Cu(pymca $)_{2} \mathrm{Br}_{\mathrm{n}}$, where pymca stands for pyrimidine-2-carboxylate [4]. The crystal belongs to the orthorhombic space group Pmc21 and consists of cationic linear chains with cis-and trans-bis(pymca) bridging ligands; the coordination being through pyrimidine nitrogens and carboxylate groups. The spin exchange antiferromagnetic coupling parameter is large, equal to $-276.1 \mathrm{~cm}^{-1}$. Compound $\mathbf{2}$ is [Cu(pmdc) $]_{\mathrm{n}}$, where pmdc stands for pyrimidine4,6-dicarboxalate and belongs to the triclinic space group $\mathrm{P}_{\overline{1}, 2}$ [5]. It was found to contain $1 \mathrm{D}[\mathrm{Cu}(\mathrm{pmdc})]$ chains. Within the chain the $\mathrm{Cu}(\mathrm{II})$ ions have square planar geometry, involving two trans coordinated oxygens and two trans coordinated nitrogen atoms of two bis-chelating pmdc ligands; the bridging metal ions are $6.0 \AA$ apart, with a quite small antiferomagnetic coupling of $-38.8 \mathrm{~cm}^{-1}$.

\section{EXPERIMENTAL}

Our theoretical work is divided into two parts:

(1) Calculations of the unit cell parameters by optimizing the geometry of the unit cell and at the same time varying the crystal parameters until the forces acting on the atoms reach a given criterion. For this procedure the quantum chemistry package QUANTUM-ESPRESSO ver. 5.2.1 was employed [6]. This package is based on a DFT approach applied to the unit cell of the solid. Several density functionals were utilized 
including the GGA functional Pbe, Pbesol, PW91 and the LDA Pz. Both plane-waves and augmented plane-waves were used.

(2) Calculations of the $J$ exchange spin coupling constant representing the exchange energy between the spin of the copper centers of the complex were done. The broken symmetry approach was employed. Although it is an old technique [7-9], but introduced recently into the ORCA program package [10]. Among three different approaches based on broken symmetry, we choose the following relation for calculating the spin exchange parameter:

$$
J=-\left(\mathrm{E}_{\text {high-spin }}-\mathrm{E}_{\text {broken sym }}\right) /(\operatorname{Smax})^{2}
$$

Since these complexes are polymeric in nature (i.e., an infinite number of copper centers interacting with each other) an approximation was used in calculating the $J$ and Landé splitting factor $\mathrm{g}$. In this approximation an isolated ion from the unit cell containing only two copper centers was used in the calculations. The environment around the atoms coordinating each copper center was preserved. The calculations utilized the DFT approach using the cc-pvdz basis set as implemented by the ORCA quantum chemistry package version 3.03 [11]. A variety of density functionals were used including B3LYP, O3LYP, BLYP, M06, PW91, BE0, revPBE, TPSSH, $\mathrm{D} 3 \mathrm{BJ}$ and B3PW.

\section{RESULTS AND DISCUSSION}

The unit cell of compound $\mathbf{1}$ is depicted in Fig. 1. The figure shows the 2D polymeric chains along the $\mathbf{a}$ and $\mathbf{c}$ directions of the unit cell. In the a direction bridging is through the carboxylate group, while in the $c$ direction bridging is through the bromine ions. Suarez-Varela et al. [4] argued that the exchange coupling between the copper centers in the $\mathrm{c}$ axis is weak. This is due to the long $\mathrm{Cu}-\mathrm{Br}$ distance. Therefore we have to consider only the coupling in the a axis for compound $\mathbf{1}$. The unit cell of compound $\mathbf{2}$ is depicted in Fig. 2. Using the software of QUANTUM-EXPRESSO ver. 5.2.1, we were able to calculate the crystal parameters of the unit cells of compounds $\mathbf{1}$ and $\mathbf{2}$. Variation of the crystal parameters was done keeping the volume of the unit cell fixed. Table-1 gives a summary of the calculated parameters, obtained using different density functionals. The projector augmented waves [12] and the norm conserving [13] approaches were employed.

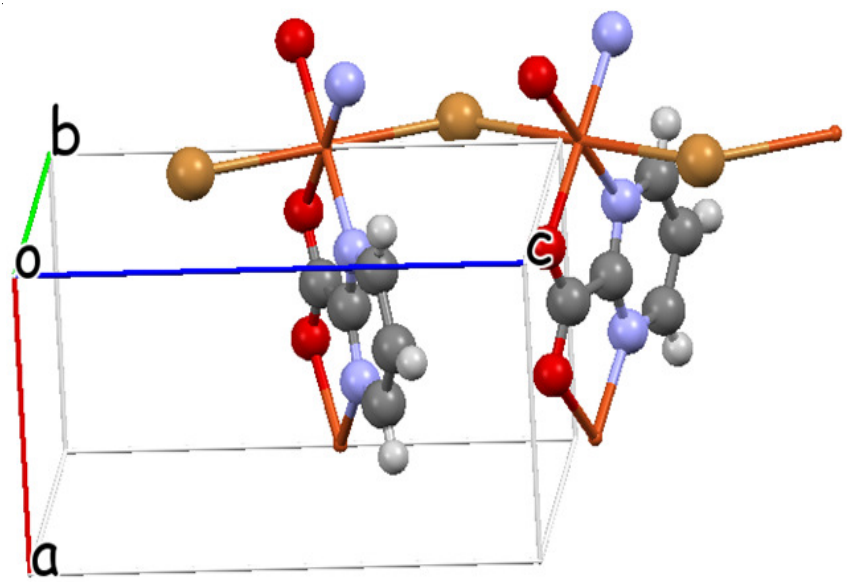

Fig. 1. Unit cell of compound $\mathbf{1}$ drawn by Mercury software [Ref. 14]

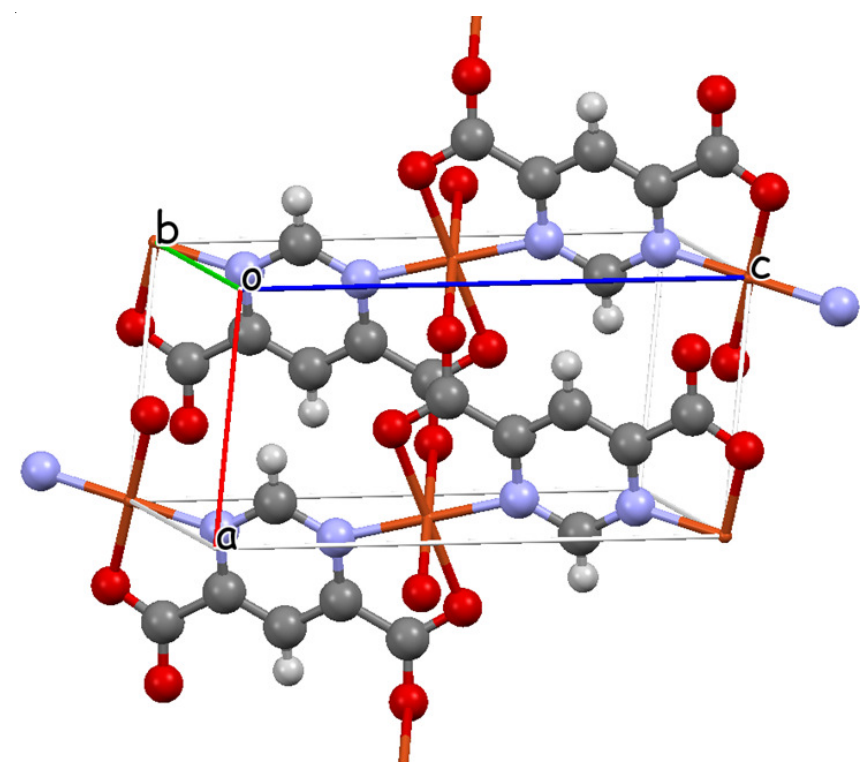

Fig. 2. Unit cell of compound 2

TABLE-1

CALCULATED CRYSTAL PARAMETERS OF COMPOUND 1

\begin{tabular}{lcccccc}
\hline \multicolumn{1}{c}{ Method } & $\mathrm{a}$ & $\mathrm{b}$ & $\mathrm{c}$ & $\alpha$ & $\beta$ & $\gamma$ \\
\hline Experimental & 5.2929 & 6.7384 & 9.8478 & 90 & 90 & 90 \\
Pbe paw* & 5.9845 & 6.1724 & 9.5083 & & & \\
Pbe NC** & 5.9687 & 6.2717 & 9.3825 & & & \\
Pbesol paw & 5.9321 & 6.2888 & 9.4148 & & & \\
Pbesol NC & 5.9249 & 6.3207 & 9.3787 & & & \\
Pw91 NC & 5.9329 & 6.2766 & 9.43174 & & & \\
Pz paw & 6.0245 & 6.5874 & 9.5843 & & & \\
\hline
\end{tabular}

*Projector augmented wave; **Pseudo potential norm conserving

For a comparative study it is of interest to find the best method that gives optimized geometry closest the experimental data; the mean bond length deviation was used as the criterion and was defined by:

$$
\mathrm{m}=J>\mathrm{i} \sum \mathrm{i}, \mathrm{j} \mid \mathrm{R}_{\mathrm{ij}}(\mathrm{x}-\mathrm{ray})-\mathrm{R}_{\mathrm{ij}} \text { (calculated) } \mid / \mathrm{n}(\mathrm{n}-1)
$$

Here, the summation goes over all atom pairs in the unit cell and $\mathrm{n}=$ number of atoms. This summation implicitly includes the bond angles. Applying this equation to compound 1, we found that the least deviation was found using Pbesol NC, which gave an error of $0.1303 \AA$. This indicates implicity that the best crystal parameters given by pbe paw. These values are $\mathbf{a}=5.9249 \AA, \mathbf{b}=6.3207 \AA$ and $\mathbf{c}=9.3787 \AA$. These values compare well with experiment and favour the use of Pbesol [15]. Table-2 summarizes the calculated crystal parameters of compound $\mathbf{2}$. In order to find the most accurate calculated parameters, eqn. 2 was applied to the cartesian coordinates of all the atoms in the unit cell. The Pbe paw functional gave the best values of the unit cell parameters, with a deviation of $0.2421 \AA$. The crystal parameters have the values $\mathbf{a}=4.7396$ $\AA, \mathbf{b}=6.8086 \AA, \mathbf{c}=10.7464 \AA, \alpha=82.8^{\circ}, \beta=96.8^{\circ}, \gamma=$ $101.1^{\circ}$. Compared to the experimental values, these parameters are not as good as those found for compound $\mathbf{1}$, this may be due to the nature of the triclinic space group of compound $\mathbf{2}$.

The best deviations calculated from eqn. 2 for our compounds are quite reasonable, because this equation includes not only bond distances but also all separations between atom pairs, including those atoms which are far apart. 
TABLE-2

CALCULATED CRYSTAL PARAMETERS OF COMPOUND 2

\begin{tabular}{lcccccc}
\hline \multicolumn{1}{c}{ Method } & $\mathrm{a}$ & $\mathrm{b}$ & $\mathrm{c}$ & $\alpha$ & $\beta$ & $\gamma$ \\
\hline Experimental & 5.1495 & 6.7207 & 10.380 & 86.5 & 100 & 108 \\
Pbe paw & 4.7397 & 6.8085 & 10.7464 & 82.8 & 97 & 101 \\
Pbe NC & 4.7065 & 7.0330 & 15.2182 & 45.0 & 105 & 93 \\
Pbesol paw & 4.5763 & 6.9663 & 10.7670 & 81.0 & 94 & 97 \\
Pbesol NC & 4.7833 & 6.6859 & 14.9804 & 46.0 & 98 & 88 \\
Pw91 NC & 4.6503 & 6.7824 & 10.9637 & 80.7 & 96 & 99 \\
Pz paw & 4.5491 & 7.0148 & 10.7546 & 80.0 & 94 & 95 \\
\hline
\end{tabular}

$\boldsymbol{J}$ Coupling and g parameters: The magnetic susceptibility measurements of compounds $\mathbf{1}$ and $\mathbf{2}$ at different temperatures indicate their antiferromagnetic nature $[4,5]$. Since the calculation of the $J$ coupling parameters using ORCA software involves two centers at a time, we chose isolated dimers for our compounds. These are given in Figs. 3 and 4. The dimers were isolated from their unit cells while keeping the environment around the copper centers intact. For comparison, different density functionals were used in the calculations. These are given in Tables 3 and 4. According to Suarez-Varela et al. [4] the experimental magnetic susceptibility data were fit to eqn. 3 and the parameters $J$, g and $\rho$ were extracted from the fit. A least square procedure was used.

\begin{tabular}{lccc}
\multicolumn{4}{c}{ TABLE-3 } \\
\multicolumn{4}{c}{$\begin{array}{c}\text { CALCULATED J COUPLING AND } \\
\text { g PARAMETERS FOR COMPOUND 1 }\end{array}$} \\
\hline \multicolumn{1}{c}{ Method } & $J$ & $\mathrm{~g}$ & $\rho$ \\
\hline Experimental & -276.1 & 2.122 & 0.002 \\
New experimental & -138.1 & 2.122 & 0.002 \\
B3LYP & -163.0 & 2.114 & \\
BLYP & -151.6 & 2.126 & \\
Cam-B3LYP & -105.6 & - & \\
D3BJ & -174.0 & 2.166 & \\
M06 & -166.9 & 2.230 & \\
O3LYP & -237.1 & 2.106 & \\
PBE & -182.6 & 2.121 & \\
Pbe0 & -130.8 & 2.127 & \\
revPbe & -193.9 & 2.128 & \\
Tpssh & -228.1 & 2.088 & \\
\hline
\end{tabular}

\begin{tabular}{lccc}
\multicolumn{4}{c}{ TABLE-4 } \\
\multicolumn{4}{c}{$\begin{array}{c}\text { CALCULATED J COUPLING AND } \\
\text { g PARAMETERS FOR COMPOUND 2 }\end{array}$} \\
\hline \multicolumn{1}{r}{ Method } & \multicolumn{1}{c}{$\mathrm{J}$} & $\mathrm{g}$ & $\rho$ \\
\hline Experimental & -38.8 & 2.17 & 0.02 \\
B3LYP & -17.3 & 2.065 & \\
BLYP & +59.0 & - & \\
Cam-B3LYP & -23.7 & 2.033 & \\
M06 & -30.5 & 2.100 & \\
revPbe & +24.6 & 2.046 & \\
Tpssh & -234.3 & 2.056 & \\
\hline
\end{tabular}

It is worth to mention that eqn. 3 was deduced based on the numerical data made by Bonner et al. [16]. On the other hand [17] succeeded to derive another eqn. 4 that can be used to fit experimental susceptibility and from it the parameters $J$, $\mathrm{g}$ and $\rho$ can be determined as usual.

We proposed to use eqn. 4 to determined the parameter $J$, $\mathrm{g}$ and $\rho$ from the experimental suscepilities at different temperature [4].
These were reproduced from eqn. 3 . We denoted as new experimental parameters. The $J$ value $-138.1 \mathrm{~cm}^{-1}$. Table- 3 deduced based on eqn. 4 is quite different from the one found based in eqn. $1=-276.1 \mathrm{~cm}^{-1}[4]$ using the same experimental data.

The presence of two different $J$ parameters of the same experimental data shows that it is dependent on the proposed equation. Any way the second $J$ parameter for compound $\mathbf{1}$ of $-138.1 \mathrm{~cm}^{-1}$ is closer to the theoretically calculated parameters than the first one. To confirm that the two standard deviations of the theoretically calculated parameters relative to the experimental extracted values were calculated. A values of 33.38 and 16.46 were found.

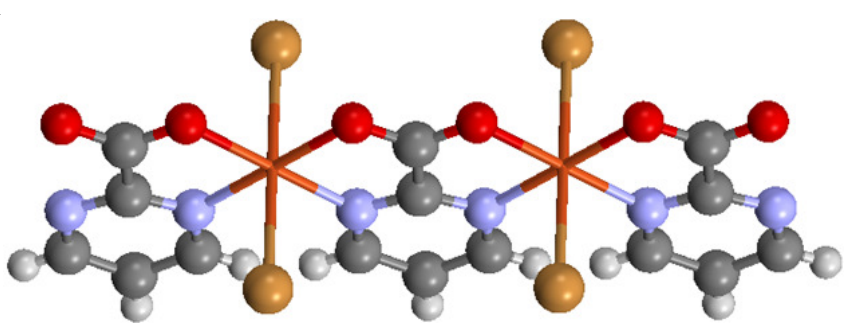

Fig. 3. Isolated ion dimer representing the bridging between two copper centres of the type O-C-O in compound (1)

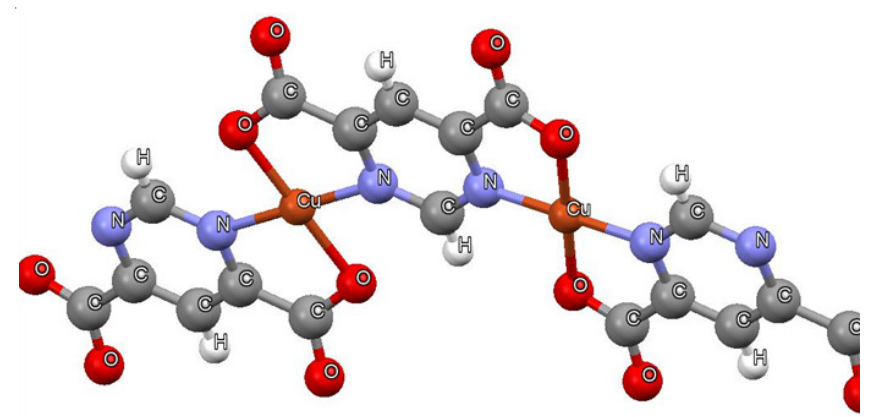

Fig. 4. Isolated ion dimer representing the bridging of two centers of copper through the $\mathrm{N}-\mathrm{CH}-\mathrm{N}$ bridge in compound 2

For compound 2, the calculated $J$ parameters are in the same range as the experimental values [5] for B3LYP, CamB3LYP and M06 functionals, preserving the antiferromagnetic character. Although BLYP and revPBE functionals show ferromagnetic trend.

We found that the calculated $J$ parameters for the different methods are on the average proportional to the value of the $\alpha$-spin densities on the bridging atoms. For example the calculated parameters of compound $\mathbf{1}$ are roughly proportional to the spin densities of the oxygen atoms of the carboxylate group. This is illustrated in Fig. 5.

$$
\begin{aligned}
& \chi_{\mathrm{A}}(1)=\left(\mathrm{Ng}^{2} \beta^{2} / \mathrm{KT}\right)(1-\rho)(0.25+0.074975 \mathrm{x}+ \\
& \left.0.075235 \mathrm{x}^{2}\right) /\left(1.0+0.9931 \mathrm{x}+0.172135 \mathrm{x}^{2}+\right. \\
& \left.0.757825 \mathrm{x}^{3}\right)+\left(\rho \mathrm{Ng}^{2} \beta^{2} / 2 \mathrm{KT}\right) \\
& \chi_{\mathrm{A}}(2)=\left(\mathrm{Ng}^{2} \beta^{2} / \mathrm{KT}\right)(1-\rho)(0.25+0.14995 \mathrm{x}+ \\
& \left.0.30094 \mathrm{x}^{2}\right) /\left(1.0+1.9862 \mathrm{x}+0.68854 \mathrm{x}^{2}+\right. \\
& \left.6.0626 \mathrm{x}^{3}\right)+\left(\rho \mathrm{Ng}^{2} \beta^{2} / 2 \mathrm{KT}\right)
\end{aligned}
$$

where $\chi_{\mathrm{A}}$ is the magnetic susceptibility the last term in these equations was added to account for the paramagnetic impurities In the sample. Where $\rho$ is the fraction of these impurities. 


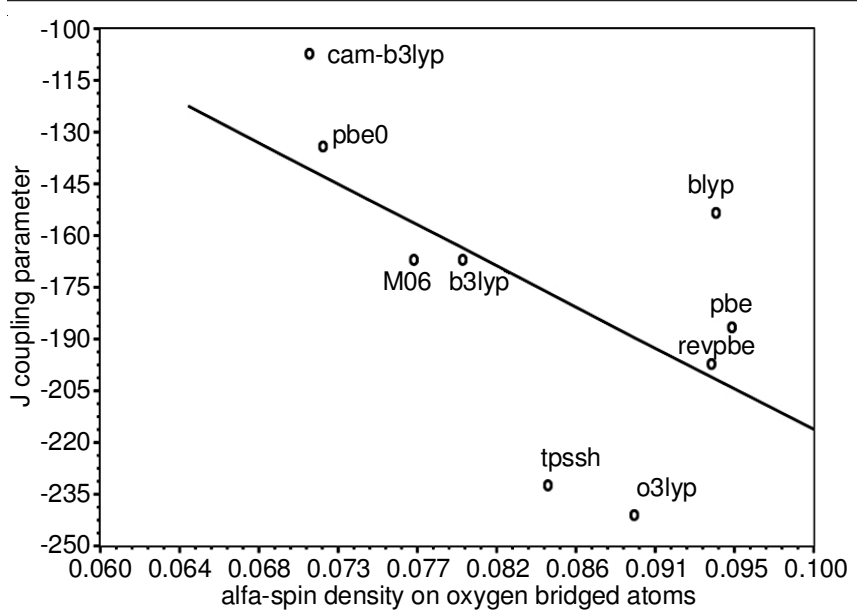

Fig. 5. A plot of $J$ coupling parameters versus spin density for compound 1

\section{Conclusion}

In this work, crystal parameters have been calculated theoretically by the DFT method. Two copper complexes were discussed using different density functionals. Relatively good results were found, with the best values given by the Pbesol functional using norm conserving pseudo-potential and the Pbe functional using projector augmented waves.

Exchange $J$ coupling parameters and Landé g parameters were also calculated theoretically. Ten and six density functional were utilized for compounds $\mathbf{1}$ and $\mathbf{2}$, respectively.

The calculated values are in quite good agreement with the experimental results. It was found that the calculated $J$ values using different methods are proportional to the $\alpha$-spin density of the bridging atoms.

\section{REFERENCES}

1. K. Nakayama, M. Nakagawa, T. Ishida, Y. Ishikawa, M. Yasui, F. Iwasaki and T. Nogami, Mol. Crysts. Liq. Crysts. Sci. Technol., 306, 379 (1997); https://doi.org/10.1080/10587259708044590.

2. Z.N. Chen, J. Qiu, W.X. Tang and K.B. Yu, Acta Crystallogr. C, 51, 1809 (1995); https://doi.org/10.1107/S0108270195002782.
3. M. Yasui, Y. Ishikawa, N. Akiyama, T. Ishida, T. Nogami and F. Iwasaki, Acta Crystallogr. B, 57, 288 (2001); https://doi.org/10.1107/S0108768101002737.

4. J. Suarez-Varela, A.J. Mota, H. Aouryaghal, J. Cano, A. Rodríguez-Diéguez, D. Luneau and E. Colacio, J. Inorg. Chem., 47, 8143 (2008); https://doi.org/10.1021/ic800625w.

5. N. Masciocchi, S. Galli, G. Tagliabue, A. Sironi, O. Castillo, A. Luque, G. Beobide, W. Wang, M.A. Romero, E. Barea and J.A.R. Navarro, Inorg. Chem., 48, 3087 (2009); https://doi.org/10.1021/ic802365w.

6. P. Giannozzi, S. Baroni, N. Bonini, M. Calandra, R. Car, C. Cavazzoni, D. Ceresoli, G.L. Chiarotti, M. Cococcioni, I. Dabo, A. Dal Corso, S. de Gironcoli, S. Fabris, G. Fratesi, R. Gebauer, U. Gerstmann, C. Gougoussis, A. Kokalj, M. Lazzeri, L. Martin-Samos, N. Marzari, F. Mauri, R. Mazzarello, S. Paolini, A. Pasquarello, L. Paulatto, C. Sbraccia, S. Scandolo, G. Sclauzero, A.P. Seitsonen, A. Smogunov, P. Umari and R.M. Wentzcovitch, J. Phys. Condens. Matter, 21, 395502 (2009); https://doi.org/10.1088/0953-8984/21/39/395502.

7. E.R. Noodleman and L. Davidson, Chem. Phys., 109, 131 (1986); https://doi.org/10.1016/0301-0104(86)80192-6.

8. A.P. Ginsberg, J. Am. Chem. Soc., 102, 111 (1980); https://doi.org/10.1021/ja00521a020.

9. T. Soda, Y. Kitagawa, T. Onishi, Y. Takano, Y. Shigeta, H. Nagao, Y. Yoshioka and K. Yamaguchi, Chem. Phys. Lett., 319, 223 (2000); https://doi.org/10.1016/S0009-2614(00)00166-4.

10. F. Neese, J. Phys. Chem. Solids, 65, 781 (2004); https://doi.org/10.1016/j.jpcs.2003.11.015.

11. Neese F, Wenmohs F. (2015) Orca version 3.03-An ab initio, DFT and Semiempirical SCF-MO package.

12. P.E. Blochl, Phys. Rev. B, 50, 17953 (1994); https://doi.org/10.1103/PhysRevB.50.17953.

13. A.M. Rappe, K.M. Rabe, E. Kaxiras and J.D. Joannopoulos, Phys. Rev. B, 41, 1227 (1990); https://doi.org/10.1103/PhysRevB.41.1227.

14. C.F. Macrae, P.R. Edgington, P. McCabe, E. Pidcock, G.P. Shields, R. Taylor, M. Towler and J. van de Streek, J. Appl. Cryst., 39, 453 (2006); https://doi.org/10.1107/S002188980600731X

15. J.P. Perdew, A. Ruzsinszky, G.I. Csonka, O.A. Vydrov, G.E. Scuseria, L.A. Constantin, X. Zhou and K. Burke, Phys. Rev. Lett., 100, 136406 (2008); https://doi.org/10.1103/PhysRevLett.100.136406.

16. J.C. Bonner and M.E. Fisher, Phys. Rev., 135, A640 (1964); https://doi.org/10.1103/PhysRev.135.A640.

17. W.E. Hatfield, R.R. Weller and J.W. Hall, Inorg. Chem., 19, 3825 (1980); https://doi.org/10.1021/ic50214a047. 